\title{
METHODS OF XS DATA PREPARATION FOR GEOMETRY WITH FUEL DUMMY
}

\author{
PAVEL SUK \\ Czech Technical University in Prague, Faculty of Nuclear Science and Physical Engineering, Department of \\ Nuclear Reactors, V Holešovičkách 2, 18000 Prague 8, Czech Republic \\ correspondence: sukpave2@fjfi.cvut.cz
}

\begin{abstract}
D deterministic core calculation represents important category of the nuclear fuel cycle and safe Nuclear Power Plant operation. The appropriate solution was not published yet. Data preparation process for non-fuel elements of the core represents the challenge for scientists. This report briefly introduce the problem of the data preparation process and gives the information about new input format for macrocode PARCS (PMAXS). The best homogenization process approach is to prepare data in infinite lattice cell for fuel assemblies, which are placed next to the another fuel assembly. Data for fuel assembly located next to the non-fuel region are better with preparation in the real geometry with the real boundary conditions. Results of the neutron spectra study show that the PMAXS file format is well prepared for the 2 group calculation, but it is not well prepared for the multigroup calculations, however the XSEC file format still gave reasonable results.
\end{abstract}

KEYwords: Eigenvalue, flux, fuel dummy, homogenization, SCALE, Serpent, PARCS.

\section{DATA PREPARATION PROCESS AND REFLECTOR CALCULATION}

Nuclear Power Plant (NPP) cores are in general too large to calculate them with exact approach of the transport equation in acceptable calculation time. Based on this fact, the data preparation process and full core calculation via deterministic macrocodes are still remaining comprehensive parts of the fuel loading patterns preparation. These two processes are, in general, connected together, but in the reality, the complexity of this problem is replaced by the easier and faster approach. This approach is based on the separation of the data preparation process for each part of the core and 3D full core deterministic calculation.

The research reactor cores are sometimes also calculated with the simplified methods. Especially the fuel loading pattern in the high power research reactors can be designed via simplified homogenization approach. The total neutron-termohydraulic solvers are also based on this methodology. Therefore the homogenization process is also important during the analysis of research reactors.

The data preparation process for the fuel elements is historically based on the exact transport simulation of the fuel assembly with reflective boundary conditions [1]. The main complication of this approach is fact that the fuel assemblies are not in the infinite medium environment during the $3 \mathrm{D}$ calculation. The reactor core does not consist of the same fuel assemblies, some flux tilt inside the fuel assemblies can be presented. Some advantages of this approach can be found in the rehomogenization process, which is described in papers [2] or [3]. The research reactors and
NPPs are almost always operated in the critical state, but during the data preparation process, the infinite medium of the the fuel assemblies can be sub-critical or super-critical in case of their enrichment and geometry. Some solution of these issues can be found in the B1 approximation, described bellow or in the article [1. The comparison of B1, P1 and CASMO methods can be found in the paper [1].

The data preparation process for non-fuel elements represents more challenge problem. The optimal solution of that problem has not been found yet, however some approaches are developed for data preparation. The main complication of the data preparation process for the non-fuel elements is based on the non-leakage medium and impossibility to calculate exact neutron spectrum during the data preparation process. The most codes are based on the diffusion solver, but the diffusion theory is not met near the boundaries [4].

Data for non-fuel regions, or reflector regions can be prepared by various approaches. For instance 1D approach or more dimension approach [5]. For better criticality and power distribution prediction, the macroscopic data are sometimes optimized. The main complication of this approach is in the high calculation time. Next complication of the fuel loading pattern optimization is in the uniqueness of the pattern. The optimization should to be made for each pattern separately. The optimization method can be found, for instance, in the paper [6].

Numerous details of data preparation for nodal calculation codes can be found also in the paper [7]. The author explain complications of the B1 method, diffusion coefficient calculation, and spatial homogenization for data preparation process there. 


\subsection{MaCROSCOPIC CROSS SECTION PREPARATION}

Macroscopic cross sections (XS) are, in general, quantities which provide information about the influence of that part of the core to the calculation. XS are defined in simple approach:

$$
\Sigma_{i, \mathrm{~g}, \mathrm{r}}=N_{i} \cdot \sigma_{i, \mathrm{~g}, \mathrm{r}},
$$

where $N_{i}$ is atomic density of the region $i$ and $\sigma_{i, \mathrm{~g}, \mathrm{r}}$ is microscopic cross section of region $i$, energy group g, and reaction $\mathrm{r}$. The more rigorous approach is based on the type of the calculation code.

The lattice codes can be divided into the discrete energy codes and continuous energy codes. In these two cathegories, the macroscopic cross sections are generated:

$$
\Sigma_{i, \mathrm{~g}, \mathrm{r}}=\frac{\sum_{h=E_{\mathrm{g}, \min }}^{E_{\mathrm{g} \max }} N_{i} \cdot \sigma_{i, h, \mathrm{r}} \cdot \Phi_{i, h}}{\sum_{h=E_{\mathrm{g}, \min }}^{E_{\mathrm{g}, \max }} \Phi_{i, h}},
$$

for lattice codes which calculated with the discrete energies. $\Phi_{i, \mathrm{~h}}$ is the neutron flux inside region $i$ and energy group $h$. For the continuous energy calculation codes, the macroscopic cross sections are calculated via:

$$
\Sigma_{i, \mathrm{~g}, \mathrm{r}}=\frac{\int_{E_{\mathrm{g}, \min }}^{E_{\mathrm{g} \max }} N_{i} \cdot \sigma_{i, \mathrm{r}}(E) \cdot \Phi_{i}(E) d E}{\int_{E_{\mathrm{g}, \min }}^{E_{\mathrm{g} \max }} \Phi_{i}(E) d E},
$$

where $\sigma_{i, \mathrm{r}}(E)$ is the microscopic cross section of the region $i$, reaction $\mathrm{r}$, and energy $E$ and $\Phi_{i}(E)$ is the neutron flux inside region $i$, energy $E$.

\subsection{B1 CORRECTION}

B1 correction, also called critical spectra correction, is a method used for treating the leakage of neutrons in the fuel assemblies places in the real core pattern. As was mentioned above, the 3D deterministic calculations are based on the two level approach. The macroscopic data for fuel assemblies are prepared for the fuel assemblies in the infinite lattice of the same assemblies, thus, the neutron leakage from the fuel assembly is zero. The B1 method prepares data based on the calculation of critical spectra in the fuel assembly and due to it treats the non zero neutron leakage.

The main idea of the B1 approximation is to find appropriate buckling factor and thus appropriate neutron spectra for XS preparation. The B1 corrected homogenised XS are then prepared with the new neutron spectra.

The buckling factor adds or removes additional neutron leakage in the fuel assemblies based on the equation:

$$
\Sigma_{\mathrm{t}, \mathrm{g}} \Phi_{\mathrm{g}} \pm i B J_{\mathrm{g}}=\chi_{\mathrm{g}} \sum_{h} \Sigma_{\mathrm{f}, h} \Phi_{h}+\sum_{h} \Sigma_{\mathrm{s}, h \rightarrow \mathrm{g}}^{0} \Phi_{h},
$$

where $\Sigma_{\mathrm{t}, \mathrm{g}}$ is the total macroscopic cross section in energy group $\mathrm{g}, \Phi_{\mathrm{g}}$ is the neutron flux in energy group $\mathrm{g}, B$ is the buckling factor, $J_{\mathrm{g}}$ is the neutron current in energy group $g, \chi_{\mathrm{g}}$ is the neutron spectra in the energy group g, $\Sigma_{\mathrm{f}, h}$ is the fission macroscopic cross section in energy group $h$ and $\Sigma_{\mathrm{s}, h \rightarrow \mathrm{g}}^{0}$ is the zero moment of scattering macroscopic cross section in energy group g.

The critical spectra is to find iteratively by increasing buckling factor. The first step is based on the calculation with $B^{2}=0$, the second step is based on the calculation with $B^{2}=10^{-6}$ and the next steps are calculated with extrapolated buckling factor from the previous calculation. The whole method is well described in the paper [1] and [7.

When the fuel assemblies in the infinite lattice have the eigenvalue less than 1 , the neutron leakage is decreasing, when the eigenvalue is larger than 1 , the leakage is increasing, in practical sense.

\section{Calculation CODES}

Calculation codes are essential for nuclear reactor operation. The codes can be divided into two main groups, macrocodes and microcodes (lattice codes). Macrocodes are used for the full core calculations with diffusion or simplified transport solution [8. Microcodes are used for exact full core transport calculation or data preparation for macrocodes. Microcodes can be than divided into the deterministic codes [9] and stochastic codes [10].

\section{PARCS}

PARCS calculation code is developed at Purdue University 11 for $3 \mathrm{D}$ NPP core calculations. PARCS is Purdue Advanced Neutron Core Simulator code which offers various calculation methods based on the diffusion solver or simplified transport solution (SP3). Some of the solvers are based on the finite difference methods, others are based on the nodal methods. 8

In the study presented below, the finite difference method based on the diffusion solver (FDM, NEMMG) or simplified transport solver (SP3) are analysed. All cases were calculated with PARCS v3.3.1 code version.

\section{SCALE}

SCALE is a comprehensive calculation package which contain deterministic codes (NEWT, TRITON) and a stochastic code (KENO) [9]. The deterministic codes NEWT and TRITON are commonly used for the data preparation. Both these codes are based on the solution of the transport equation in multigroup approach. XS can be prepared with actual flux spectrum or with B1 approximation. TRITON code is able to carried out burnup calculations in contrast with NEWT. All cases were calculated with SCALE 6.2.3 code version $[12]$.

\section{TEST CASE}

The model consisted of the simplified fuel assemblies and simplified fuel dummy filled with water was developed as a test case. This model is not usual, however 
it includes region for that the data preparation is complicated. The visualisation of the test case is shown in figure 1. This geometry can be found in the research reactors, where the water can be replaced by the research equipment like irradiation channels, detectors or other devices.

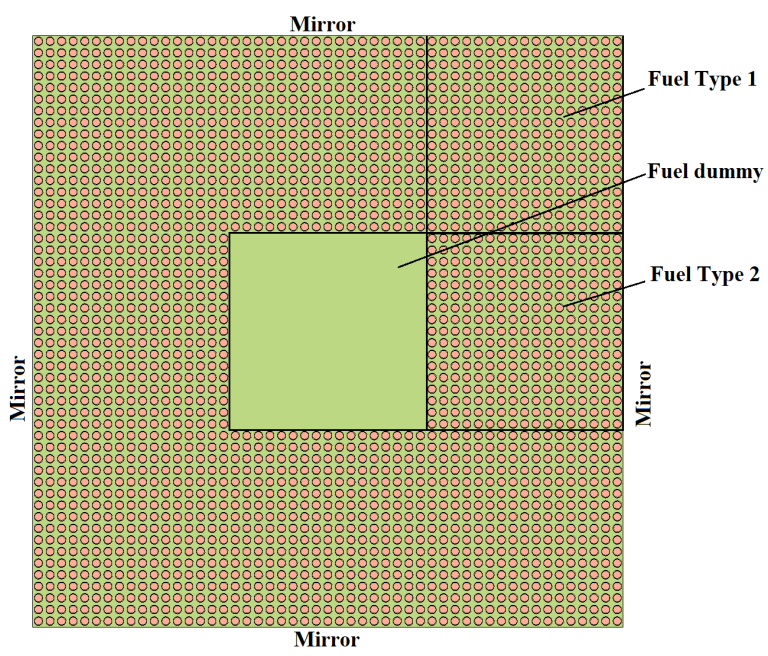

Figure 1. Visualisation of the test case model

Test case consists of the 3 elements types. The whole structure is placed in the infinite lattice of the same structures. Fuel assembly 1 (FA1) seems to be like a fuel assembly in the infinite lattice of the same assemblies, because each boundary is connected to the fuel assembly. Fuel assembly 2 (FA2) has three sides connected with the fuel assembly and one side is connected to the water fuel dummy. Water fuel dummy (DUM) is simulated with the same temperature and density conditions as the moderator inside the fuel assemblies $\left(\rho=0.71667 \mathrm{~g} / \mathrm{cm}^{3}, T=578 \mathrm{~K}\right)$.

Fuel assemblies consist of $17 \times 17$ fuel pins. Description of the material and geometry of the test case is given in table 1. Two different enrichments of the fuel pins were simulated: $0.7 \%$ - sub-critical system and $4.3 \%$ super-critical system.

\begin{tabular}{cc}
\hline \multicolumn{2}{c}{ Test case parameters } \\
\hline Fuel diameter & $0.82 \mathrm{~cm}$ \\
Cladding diameter & $0.95 \mathrm{~cm}$ \\
Pin pitch & $1.26 \mathrm{~cm}$ \\
Fuel density & $10.219 \mathrm{~g} / \mathrm{cm}^{3}$ \\
Moderator density & $0.71667 \mathrm{~g} / \mathrm{cm}^{3}$ \\
Fuel temperature & $1100 \mathrm{~K}$ \\
Cladding temperature & $600 \mathrm{~K}$ \\
Moderator temperature & $578 \mathrm{~K}$ \\
\hline
\end{tabular}

TABLE 1. Description of the material and geometry of the test case

\section{Calculation}

Many different approaches were realised during the analysis of behaviour in the test case. In the first stage, the influence of B1 approximation and noncritical flux were analysed. The next part of the study was focused on the downscatter treatment. Last part of the study deals with the multigroup approach and spectra comparison. Some of the diffusion codes are designed to use only downscattering, therefore the scattering cross sections are modified via:

$$
\hat{\Sigma}_{\mathrm{g}, \mathrm{h}}=\Sigma_{\mathrm{g}, \mathrm{h}}-\frac{\Phi_{\mathrm{h}}}{\Phi_{\mathrm{g}}} \Sigma_{\mathrm{h}, \mathrm{g}},
$$

where the $\Sigma_{\mathrm{g}, \mathrm{h}}$, respective $\Sigma_{\mathrm{h}, \mathrm{g}}$ is scattering cross section from group g to group $h$, respective from $h$ to g. $\Phi_{\mathrm{h}}$, respective $\Phi_{\mathrm{g}}$ is the neutron flux of group h, respective $\mathrm{g}$.

The compliance of the simplified solution calculated via PARCS was rated by the eigenvalue compliance, see equation (6) and the neutron flux values inside homogenised areas, see equation (7). The reference solution was obtained by the whole geometry simulation in the SCALE 6.2.3 calculation code.

$$
\begin{gathered}
\Delta k_{\mathrm{eff}}=\left(k_{\mathrm{eff}, \mathrm{SCALE}}-k_{\mathrm{eff}, \mathrm{PARCS}}\right) \cdot 10^{5} \\
\Delta \Phi_{\mathrm{g}}=\sqrt{\frac{1}{N} \sum_{i=0}^{N}\left(\frac{\Phi_{\mathrm{g}, \mathrm{SCALE}}^{i}-\Phi_{\mathrm{g}, \mathrm{PARCS}}^{i}}{\Phi_{\mathrm{g}, \mathrm{SCALE}}^{i}} 100\right)^{2}}
\end{gathered}
$$

\subsection{TWO GROUP APPROACH}

The two group XS were prepared with the SCALE TRITON calculation code in this section. The data for macrocode PARCS can be prepared by 2 ways, with PMAXS files and XSEC files. The data from SCALE TRITON calculation code was prepared by the GenPMAXS [13] program, which prepares PMAXS files for each region. The obtained results with PMAXS files showed good agreement, therefore the XSEC file format was not used during two group analyses.

Many calculation modes and homogenization approaches were carried during this analysis:

- CASE1 - Data for each different part of model was prepared by simulation in the real geometry with the real boundary conditions with B1 correction.

- CASE1_COR - CASE1 with downscatter correction via equation (5).

- CASE2 - Data for the fuel assemblies was prepared by simulation with the reflective boundary condition, data for DUM was prepared by simulation in the real geometry with the real boundary conditions with B1 correction.

- CASE2_COR - CASE2 with downscatter correction via equation (5).

- CASE3 - Data for FA1 was prepared by simulation with the reflective boundary condition, other data was prepared by simulation in the real geometry with the real boundary conditions with B1 correction. 
- CASE3 COR - CASE3 with downscatter correction via equation (5).

- CASE4 - CASE1 without B1 correction.

- CASE5 - CASE2 without B1 correction.

- CASE6 - CASE3 without B1 correction.

- CASE7 - Data for fuel assemblies was prepared by the simulation with the reflective boundary condition with B1, data for DUM was prepared by the simulation in the real geometry with the real boundary conditions without B1 correction.

- CASE8 - Data for FA1 was prepared by the simulation with the reflective boundary condition with B1, data for other regions were prepared by the simulation in the real geometry with the real boundary conditions without B1 correction.

\subsection{1. $0.7 \%$ ENRICHMENT}

Fuel enriched only with $0.7 \%$ was simulated to investigate agreement for the sub-critical core regions. The results of eigenvalue calculation and relative flux differences are shown in table 2 visualised in figures 2 and 3 .

\begin{tabular}{rccc}
\hline Modes & $\Delta k_{\text {eff }}(\mathrm{pcm})$ & $\Delta \Phi_{1}(\%)$ & $\Delta \Phi_{2}(\%)$ \\
\hline CASE1 & 51.8 & 4.52 & 1.85 \\
CASE1_COR & 71.7 & 4.56 & 1.92 \\
CASE2 & 91.4 & 3.47 & 1.40 \\
CASE2_COR & -46.0 & 8.60 & 1.92 \\
CASE3 & 8.6 & 4.23 & 1.67 \\
CASE3_COR & 26.0 & 4.26 & 1.73 \\
CASE4_COR & 189.5 & 7.92 & 2.61 \\
CASE5_COR & 183.7 & 6.15 & 2.00 \\
CASE6_COR & 127.6 & 7.34 & 2.30 \\
CASE7_COR & 66.3 & 4.11 & 1.15 \\
CASE8_COR & 59.1 & 6.15 & 2.00 \\
\hline
\end{tabular}

TABle 2. Two group results of the the test case with fuel enrichment $0.7 \%$ (reference $k_{\text {eff }}=0.82853$ )

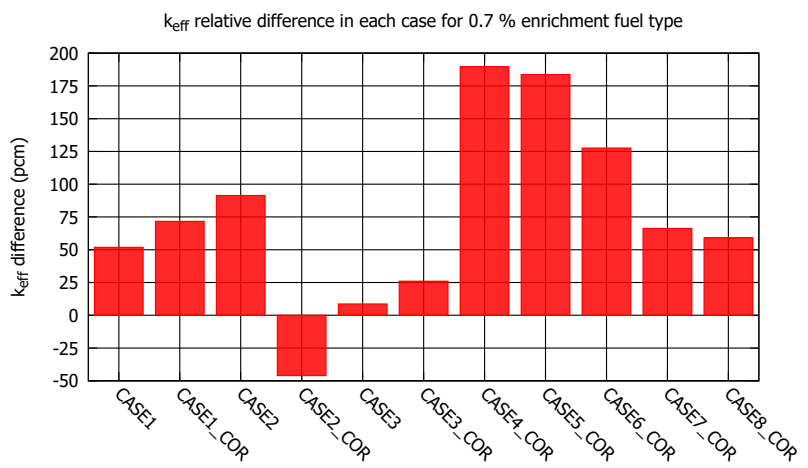

Figure 2. Relative eigenvalue differences in the calculation modes with $0.7 \%$ fuel enrichment test case

In case of eigenvalue, the CASE1, CASE2_COR, CASE3 and CASE3_COR were in the best agreement with the lattice code. On the opposite, the cases without B1 correction (CASE4, CASE5 and CASE6) were

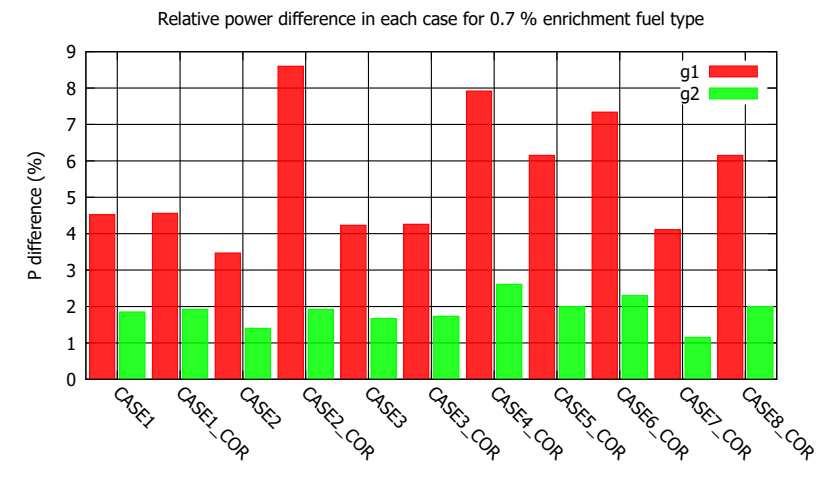

FiguRE 3. Relative power differences in the calculation modes with $0.7 \%$ fuel enrichment test case

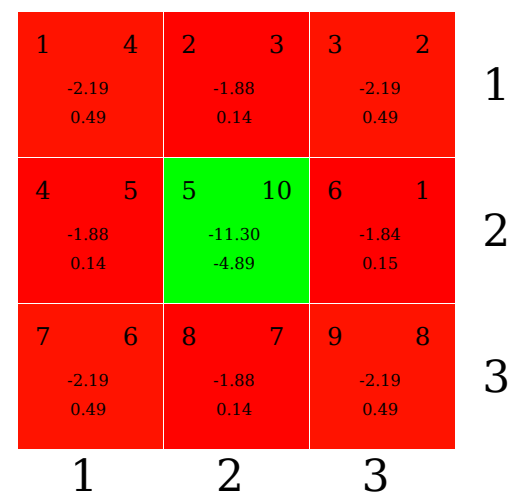

Legend

Figure 4. 2D flux differences for each part of the test case in CASE3 and enrichment $0.7 \%$

in the worse agreement. The neutron flux comparison showed that the thermal neutron group deviates around $2 \%$ in all cases, but the fast neutron group flux difference reached up to $9 \%$ in CASE2_COR, CASE4_COR and CASE6_COR.

The best results in case of eigenvalue and $\Phi_{\mathrm{g}}$ are in CASE3. 2D flux differences of CASE3 are shown in figure 4

Figure 4 shows the best agreement in the thermal group (only $0.49 \%$ ) for fuel assemblies and only $2 \%$ deviation in the fast energy group. The neutron fluxes in the fuel dummy region inside the test case were determined with worse agreement. Up to $5 \%$ for thermal energy group and up to $11.5 \%$ for the fast energy group.

\subsection{2. $4.3 \%$ ENRICHMENT}

The nuclear fuel in the NPP cores is in general enriched up to $5 \%$. The fuel pellets with enrichment $4.3 \%$ are usually in the active length of the fuel pins and, moreover, they make the simulated system supercritical.

The results of the eigenvalue and the relative flux differences are shown in the table 3, visualised in figures 5 and 6 .

The best agreement in case of eigenvalue were reached in the cases without B1 correction (CASE4_COR, CASE5_COR, and CASE6_COR). On the other hand, the fast neutron flux differences 


\begin{tabular}{rccc}
\hline Modes & $\Delta k_{\text {eff }}(\mathrm{pcm})$ & $\Delta \Phi_{1}(\%)$ & $\Delta \Phi_{2}(\%)$ \\
\hline CASE1 & 688.6 & 5.17 & 4.89 \\
CASE1_COR & 690.6 & 5.14 & 4.97 \\
CASE2 & 767.6 & 5.43 & 4.74 \\
CASE2_COR & 730.6 & 5.49 & 4.87 \\
CASE3 & 625.6 & 5.16 & 4.81 \\
CASE3_COR & 626.6 & 5.14 & 4.89 \\
CASE4_COR & 173.6 & 7.82 & 4.61 \\
CASE5_COR & 121.6 & 9.61 & 4.52 \\
CASE6_COR & 63.6 & 8.23 & 4.41 \\
CASE7_COR & 792.6 & 5.15 & 5.84 \\
CASE8_COR & 418.6 & 5.93 & 6.36 \\
\hline
\end{tabular}

TABle 3. Two group results of the test case with fuel enrichment $4.3 \%$ (reference $k_{\text {eff }}=1.32588$ )

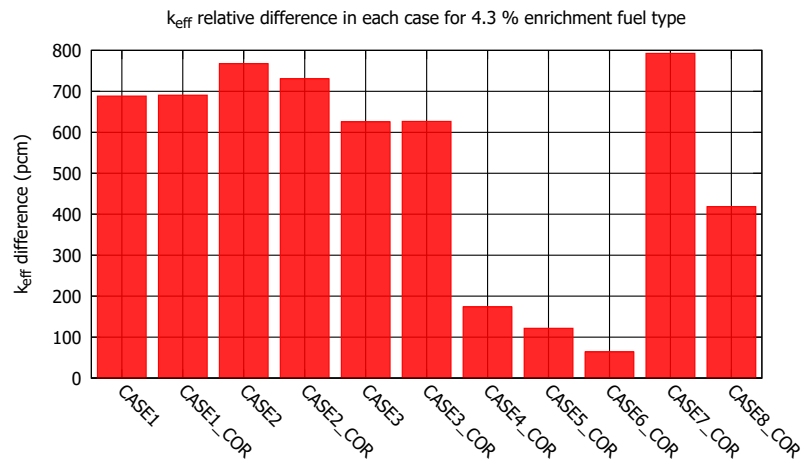

FiguRE 5. Relative eigenvalue differences in the calculation modes with $4.3 \%$ enrichment fuel test case

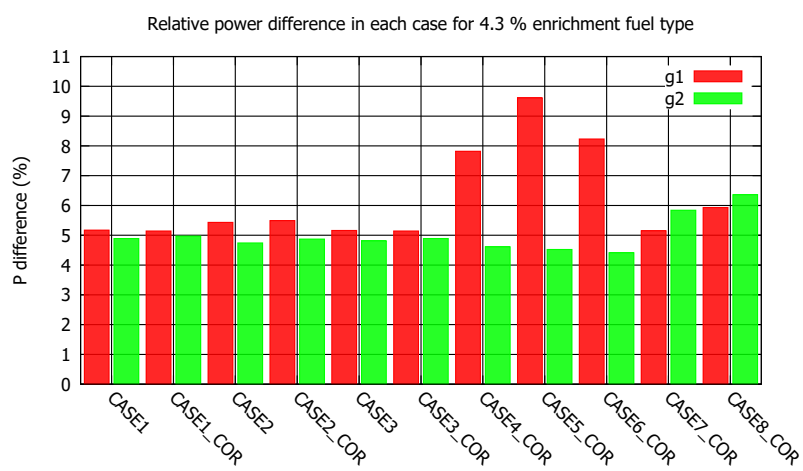

FiguRE 6. Relative power differences in the calculation modes with $4.3 \%$ enrichment fuel test case

were the highest in that three cases. The $2 \mathrm{D}$ flux differences of CASE6_COR is shown in figure 7 CASE6_COR model showed the best result in case of eigenvalue, with difference only $63 \mathrm{pcm}$.

Figure 7 shows relatively good agreement in thermal neutron flux in fuel assemblies and fast neutron flux in DUM, only up to 3\%. Fast neutron flux in fuel assemblies and thermal neutron flux in the DUM were determined with worse agreement, up to $11 \%$.

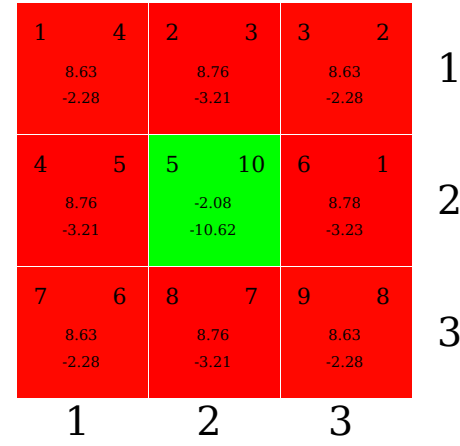

Legend

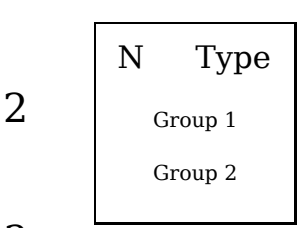

Figure 7. 2D flux differences for each part of the test case in CASE6_COR and enrichment $4.3 \%$

\subsubsection{Discussion}

The above results showed that the data preparation based on the actual boundary condition cannot bring good results, because the PARCS calculation code does not know the actual neutron leakage from the system and due to it there is lack of the informations to properly simulate the system.

The best agreement in the lower enriched case was obtained with the B1 correction. This situation corresponds with the physical solution. If the solved problem is sub-critical, the B1 approximation simulates less neutron leakage to reach criticality during the data preparation process. Because the each part of the test case is "locally" sub-critical, it means that the neutron spectra is changed in each part with the same direction and due to that the results are better with B1 approximation.

The situation in the higher enriched case is different. The whole test case is super-critical, but the water fuel dummy is "locally" sub-critical. The B1 correction increases neutron leakage from the whole system, but the water fuel dummy is "locally" sub-critical. The B1 correction changes the neutron spectra in both regions with the same direction and due to that the neutron spectra in the water fuel dummy is very different during the XS preparation. The better results were obtained without B1 correction and it also corresponds with the above theory.

\subsection{Multigroup approach}

To better understand the behaviour of the water-fuel neutron transport in the macrocodes, there was pressure to analyse and compare multigroup neutron flux in the PARCS and SCALE code systems. The fuel assembly with the mirror boundary conditions was analysed in the multi group approach.

The neutron flux is constant in the assembly with the mirror boundary condition and due to it only eigenvalue was analysed. As it was told before, PARCS calculation code offers to use two types of libraries structures (PMAXS and XSEC). The eigenvalues were calculated with many different ways:

- XSEC - XSEC without downscatter correction,

- XSEC COR - XSEC with downscatter correction, 
$\mathrm{k}_{\text {eff }}$ relative difference between SCALE and PARCS

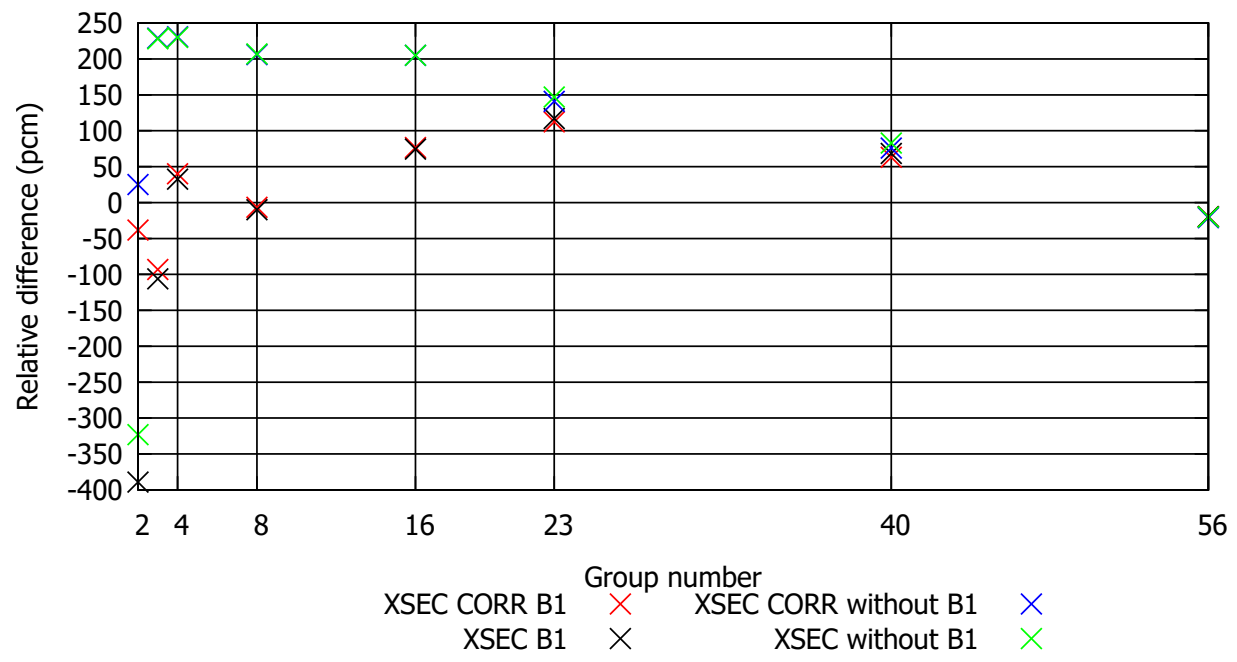

FIGURE $8 . k_{\text {eff }}$ relative deviation with XSEC input files

- PMAXS - PMAXS without downscatter correction,

- PMAXS COR - PMAXS with downscatter correction.

Results of the simulations are in table 4

The PMAXS input format brings better results in two group approach, but the large deviations from the reference solution was found in many energy group analysis. The reference solution of the test case geometry was calculated with SCALE TRITON sequence $k_{\text {eff }}=0.86041$. It seems that the PMAXS input format is well prepared for two group calculations, which are most common in the full core calculations, but it is absolutely inappropriate for multi groups calculation.

The XSEC input format seems to be more appropriate for multigroup analysis. There are only small deviations between diffusion solution and appropriate transport solution. The highest deviation with the B1 correction is in case of XSEC for two group calculation. This deviation is caused by the fact, that PARCS was used in the diffusion mode and the inputs scatter matrix is limited into the downscatter from fast to thermal group. Due to that fact, the two group solution needs to be corrected by downscattering via the equation (5). When the downscatter was corrected, the results were similar to those obtained with PMAXS COR. This situation can be seen in both simulations (with B1 and without B1 approach).

Deviations between the XSEC and XSEC COR for more group approach are smaller, because for more group calculations, there are total scatter matrix input data. Due to it there is no necessary to use correction via equation (5). Based on this fact, the XSEC without downscatter correction input format is used for other more group calculations. The relative difference of $k_{\text {eff }}$ between SCALE and PARCS for $\mathrm{XSEC}$ input format is in figure 8 .

The convergence problem with the calculation were found in case of higher group number. The calculation did not converge automatically, but after it reached maximum number of iteration, it finished and gave a result without any warning message.

The influence of B1 correction decreases with higher number of energy groups. This behaviour begins during the data preparation process by microcode SCALE TRITON, because the group structure for the data preparation process was 56 group ENDF/B-VII.0 library and microcode SCALE prepares critical spectra in 56 groups. When user wants to calculate XS with B1 leakage correction in 56 groups, the collapsing to the energy groups is independent on the neutron spectra.

\subsection{NEUTRON FLUX SPECTRA COMPARISON}

The neutron spectra was created to better understand the behaviour in the fuel regions near to the water fuel dummy region. The spectra was calculated with 56 energy groups. There was also the same convergence complications as were described in the previous sections. Because of the results of two group calculation, there was analysed only three cases without B1 leakage correction (CASE4, CASE5 and CASE6). The data for scattering were not corrected for downscattering, because the results from section 4.2 showed that the downscattering correction is not necessary.

The neutron flux spectra comparison gives the information about the spectra and slowing down in the SCALE TRITON and PARCS codes. The spectra were analysed separately in fuel regions and in the DUM region.

\subsection{1. $0.7 \%$ ENRICHMENT}

The test case with $0.7 \%$ fuel enrichment represents the sub-critical problem. The neutron spectra in the fuel assembly FA1, respective in the DUM region are in figures 9 respective 10 . The neutron flux spectra 


\begin{tabular}{ccccccccc}
\hline Groups & \multicolumn{3}{c}{ With B1 } & \multicolumn{3}{c}{ Without B1 } \\
\hline & PMAX COR & PMAX & XSEC COR & XSEC & PMAX COR & PMAX & XSEC COR & XSEC \\
2 & -38 & -47 & -38 & -389 & 25 & 25 & 25 & -323 \\
3 & -2296 & -2307 & -93 & -106 & -1950 & -1950 & 229 & 228 \\
4 & -2168 & -2174 & 40 & 33 & -1952 & -1952 & 231 & 230 \\
8 & -2192 & -2195 & -7 & -10 & -1954 & -1953 & 206 & 207 \\
16 & -2110 & -2112 & 77 & 74 & -1955 & -1955 & 205 & 205 \\
23 & -31510 & -31512 & 112 & 117 & -31479 & -31479 & 141 & 147 \\
40 & -31495 & -31496 & 63 & 68 & -31479 & -31479 & 76 & 83 \\
56 & -31985 & -31987 & -21 & -20 & -31987 & -31987 & -21 & -20 \\
\hline
\end{tabular}

TABLE $4 . k_{\text {eff }}$ relative difference between SCALE and PARCS with multigroup approach for $0.7 \%$ enriched fuel assembly with mirror boundary condition

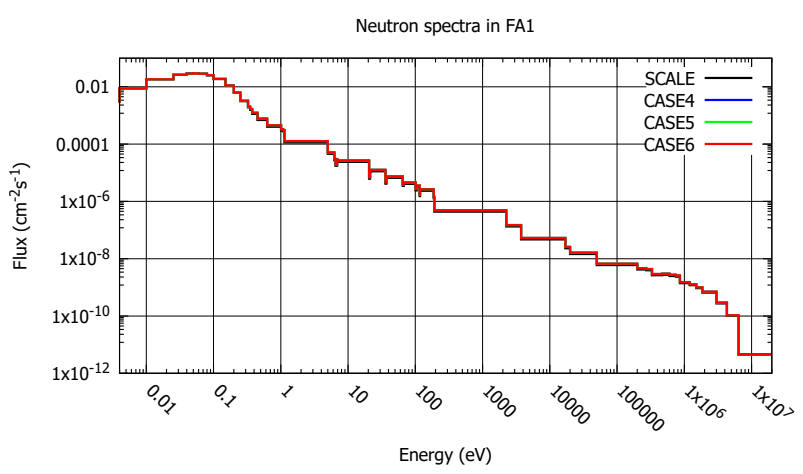

Figure 9. Neutron flux spectra in the fuel assembly FA1 with $0.7 \%$ fuel enrichment

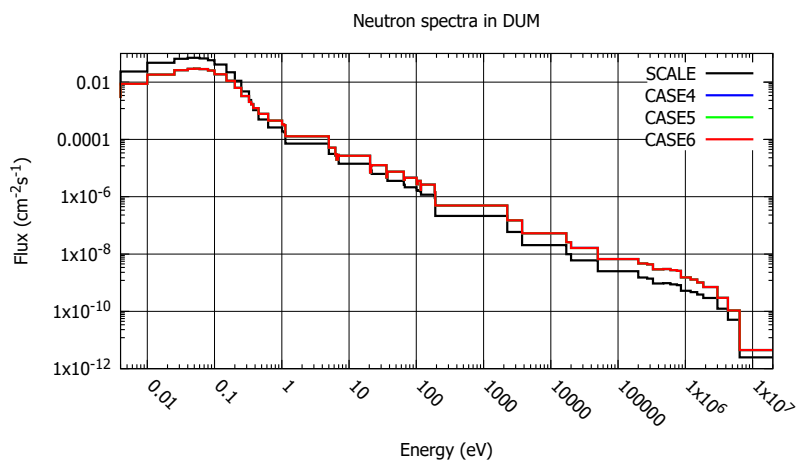

Figure 10. Neutron flux spectra in the DUM with $0.7 \%$ fuel enrichment

in FA1 and FA2 are similar and the differences are not visible in the spectra comparison. The eigenvalues and their differences from the reference calculation are in table 5

Main result from figures 9 and 10 is that the fuel spectrum in the fuel assemblies is well calculated with the PARCS calculation code.

Differences between the PARCS and SCALE calculation were analysed via equation:

$$
\Delta \Phi_{\mathrm{g}}=\frac{\Phi_{\mathrm{g}}^{\mathrm{SCALE}}-\Phi_{\mathrm{g}}^{\mathrm{PARCS}}}{\Phi_{\mathrm{g}}^{\mathrm{SCALE}}}
$$

The neutron spectra differences in figures 11,12

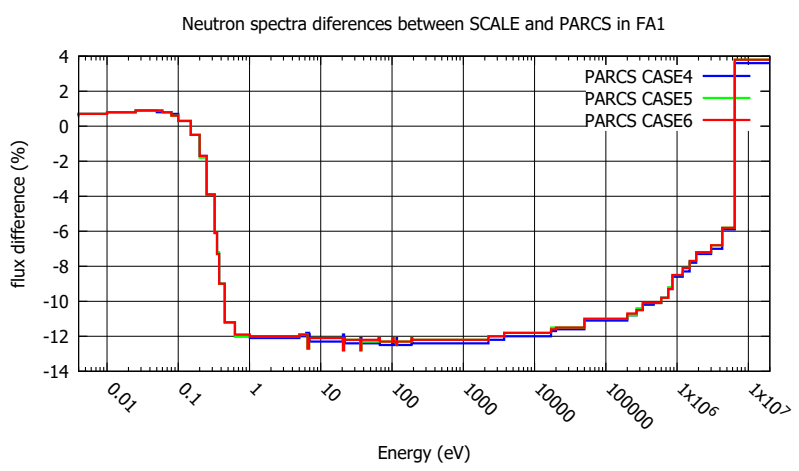

FiguRE 11. Relative difference in spectra between SCALE and PARCS calculation for FA1 for $0.7 \%$ fuel enrichment

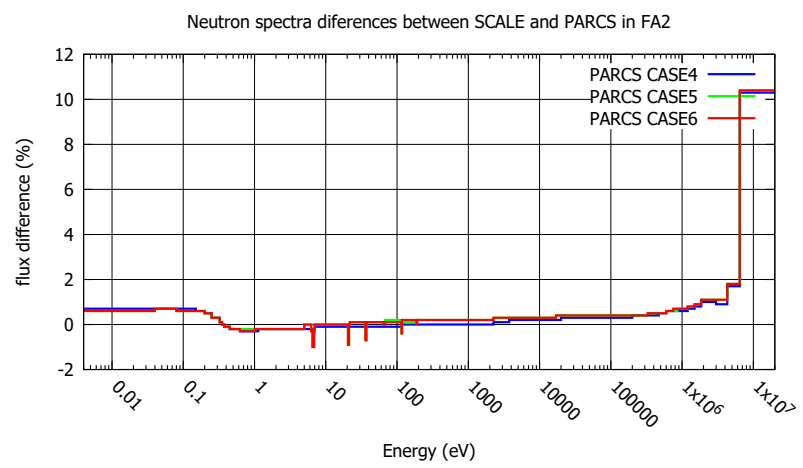

FiguRE 12. Relative difference in spectra between SCALE and PARCS calculation for FA2 for $0.7 \%$ fuel enrichment

and 13 show that the spectrum calculated in the FA1 has the large deviations in the slowing down region and particularly in the high energy neutron region. The FA2 has great deviations only in the high energy neutron region. In the fuel assemblies, the relative deviations are only up to $12 \%$ in the absolute values, but the deviations in the DUM region are up to $225 \%$. That means that the data prepared for the DUM region are not prepared well and the behaviour of neutrons near the DUM region is not described correctly. 


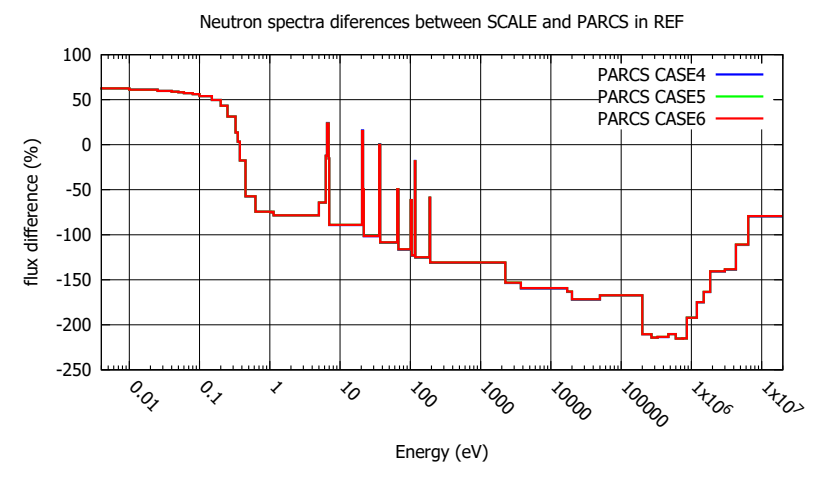

FIGURE 13. Relative difference in spectra between SCALE and PARCS calculation for DUM for $0.7 \%$ fuel enrichment

\subsection{2. $4.3 \%$ ENRICHMENT}

Fuel pins with $4.3 \%$ enrichment represent the supercritical problem, which, based on the information obtained during the two group study, can brings more deviated results. The eigenvalue differences are in table 5 The neutron spectra were similar to the neutron spectra in the lower enrichment case with little bit higher discrepancies.

\begin{tabular}{|c|c|c|}
\hline Mode & $\Delta k_{\text {eff }} 0.7 \%(\mathrm{pcm})$ & $\Delta k_{\mathrm{eff}} 4.3 \%(\mathrm{pcm})$ \\
\hline CASE4 & 120 & 923 \\
\hline CASE5 & 24 & 783 \\
\hline CASE6 & 61 & 837 \\
\hline
\end{tabular}

TABLE $5 . k_{\text {eff }}$ relative difference between SCALE and PARCS with 56 groups calculation

\subsection{SP3 MULTIGROUP SOLUTION}

The PARCS calculation code is able to calculate simplified transport approach (SP3) instead of the diffusion approach. The NEMMG solver with the nspn 3 was used to calculate the test geometry.

The only appropriate approach of CASE4, CASE5 and CASE6 were calculated. The results of the eigenvalue for both enrichment cases are in table 6 .

\begin{tabular}{|c|c|c|}
\hline Mode & $\Delta k_{\text {eff }} 0.7 \%(\mathrm{pcm})$ & $\Delta k_{\mathrm{eff}} 4.3 \%(\mathrm{pcm})$ \\
\hline CASE4 & -52 & 732 \\
\hline CASE5 & -148 & 590 \\
\hline CASE6 & -111 & 646 \\
\hline
\end{tabular}

TABLE $6 . k_{\text {eff }}$ relative difference between SCALE and PARCS with 56 groups calculation and SP3

The neutron spectra for SP3 calculations are very similar to the neutron spectra calculated with diffusion approach. The main difference is in the higher energies in the FA2 fuel assembly with $0.7 \%$ enrichment. The neutron spectra are more accurate in the higher energies in comparison with the diffusion approach for higher enriched fuel. The eigenvalue deviations increased in comparison to the diffusion approach in lower enrichment case, but deviation decreased with higher enrichment case.

\section{Conclusion}

Various different approaches were analysed in this paper. Two different enrichments were studied. Results of the two group calculation showed that in case of lower enrichment, the data prepared by the simulation of the FA1 fuel assembly in the infinite lattice and FA2 and DUM in the real geometry with B1 approximation (CASE3) well reproduce the eigenvalue. The relative flux differences were also calculated with the smallest difference in the CASE3. On the other hand, the cases, which were calculated without B1 approximations gave more-times worse results in case of lower enrichment. The results were opposite in the case of higher enrichment than in the lower enrichment calculation. The best agreement were obtained during the calculation without B1 approximation and the same data preparation scheme (CASE6). The eigenvalue was bellow 100 pcm from reference calculation, but the fast group neutron fluxes were calculated with higher difference (up to $10 \%$ ) in each calculation without B1 approximation.

The upper results are consistent with B1 correction. In the case of lower enrichment, the situation corresponds with the physical solution. The whole geometry is sub-critical and B1 approximation decreases the neutron leakage to reach criticality during the data preparation process. In case of higher enrichment, the whole geometry is super-critical, but there is part which is "locally" sub-critical - DUM. The B1 approximation increases neutron leakage from the system to reach the criticality, but this does not correspond with the physical solution in the DUM part. The neutron spectra is changed there and due to that the B1 correction cannot brings better results in the case of higher enrichment.

The main objective of the spectra study is that PMAXS files, which PARCS use newly, are not able to calculate core with multigroup approach. The eigenvalue differences showed that there is any problem with data preparation for that calculation. The results of calculations with XSEC file format bring reasonably results of eigenvalue despite the convergence problems.

Based on the results of multigroup study, the neutron spectra in multigroup approach were calculated for test cases without B1 approximation. Calculations without B1 approximation were chosen because the final geometry was not critical and there are still unsolved problems with B1 approximation. The paper [7] informs that the $\mathrm{B} 1$ calculation is not rigorous and due to the reaching critical spectra by the changing absorption in each energy group, the result are not representative for LWR calculations, where the criticality is reached by the absorption of only thermal neutrons.

The neutron spectra in the fuel assemblies were determined with a good agreement with the reference 
calculation, but the spectra in the DUM was determined with larger discrepancies. It is well known, that the diffusion theory is not fulfilled in the water fuel dummy region and the discrepancies are mostly based on this fact.

There are also methods, which can bring better results, for instance the influence of neutron scatter anisotropy in the DUM. The different approach of the DUM preparation data will be study in the future.

\section{ACKNOWLEDGEMENT}

This work was supported by the project no. CZ.02.1.01/0.0/0.0/16_013/0001790 supported by Operational Programme Research, Development and Education co-financed from European Structural and Investment Funds and from state budget of the Czech Republic and it was supported by the Grant Agency of the Czech Technical University in Prague, grant no. SGS19/114/OHK4/2T/14.

\section{LIST OF SYMBOLS}

$\Phi_{i}(E)$ Neutron flux of region $i$ and energy $E\left[\mathrm{~cm}^{-2} \mathrm{~s}^{-1}\right]$

$\Phi_{i, \mathrm{~g}} \quad$ Neutron flux of region $i$ and energy group $\mathrm{g}$ $\left[\mathrm{cm}^{-2} \mathrm{~s}^{-1}\right]$

$\sigma_{i, \mathrm{~g}}$ Microscopic cross section of region $i$ and group g $\left[\mathrm{cm}^{2}\right]$

$\sigma_{i}(E)$ Microscopic cross section of region $i$ and energy $E$ $\left[\mathrm{cm}^{2}\right]$

$\Sigma_{i, \mathrm{~g}}$ Macroscopic cross section of region $i$ and group g $\left[\mathrm{cm}^{-1}\right]$

$N_{i} \quad$ Atomic density of region $i\left[\mathrm{~cm}^{-3}\right]$

$E_{\mathrm{g}, \text { min }}$ Minimal energy of boundary related to the energy group g $[\mathrm{eV}]$

$E_{\mathrm{g}, \max }$ Maximal energy of boundary related to the energy group g $[\mathrm{eV}]$

$N$ Number of regions in the test case $[-]$

$\Sigma_{\mathrm{t}, \mathrm{g}}$ Total macroscopic cross section for energy group g $\left[\mathrm{cm}^{-1}\right]$

$B$ Buckling factor $\left[\mathrm{cm}^{-1}\right]$

$J_{\mathrm{g}} \quad$ Neutron current in group $\mathrm{g}\left[\mathrm{cm}^{-2} \mathrm{~s}^{-1}\right]$

$\chi_{\mathrm{g}} \quad$ Neutron spectra in group $\mathrm{g}[-]$

$\Sigma_{\mathrm{f}, h} \quad$ Fission macroscopic cross section $\left[\mathrm{cm}^{-1}\right]$

$\Sigma_{\mathrm{s}, h \rightarrow \mathrm{g}}^{0}$ Scattering macroscopic cross section $\left[\mathrm{cm}^{-1}\right]$

$k_{\text {eff,X }}$ Eigenvalue calculated with mode X [-]

$\Delta k_{\text {eff }}$ Difference of the eigenvalue [pcm]

$\Delta \Phi_{\mathrm{g}}$ Relative difference of $\Phi_{\mathrm{g}}$ for the whole test case [\%]

\section{REFERENCES}

[1] S. Choi, K. S. Smith, H. Kim, et al. On the diffusion coefficient calculation in two-step light water reactor core analysis. Journal of Nuclear Science and Technology 54(6):705-715, 2017. DOI:10.1080/00223131.2017.1299648

[2] A. Dall'Osso. A spatial rehomogenization method in nodal calculations. Annals of Nuclear Energy 33:869-877, 2006. DOI:10.1016/j.anucene.2006.05.008
[3] P. Suk. Advanced homogenization methods for pressurized water reactors. Acta Polytechnica $C T U$ Proceedings 19:14, 2018. DOI:10.14311/APP.2018.19.0014.

[4] P. Reus. Neutron Physics. 2008. ISBN: 2759800415.

[5] K. I Usheva, S. Kutsen, A. A Khruschinsky, L. Babicheu. Generation of xs library for the reflector of vver reactor core using monte carlo code serpent. Journal of Physics: Conference Series 781:012029, 2017. DOI:10.1088/1742-6596/781/1/012029.

[6] T. Clerc, A. Hébert, H. Leroyer, et al. An advanced computational scheme for the optimization of $2 \mathrm{~d}$ radial reflector calculations in pressurized water reactors. Nuclear Engineering and Design 273:560 - 575, 2014. DOI:10.1016/j.nucengdes.2014.03.044

[7] K. S. Smith. Nodal diffusion methods and lattice physics data in lwr analyses: Understanding numerous subtle details. Progress in Nuclear Energy 101:360 369, 2017. Special Issue on the Physics of Reactors International Conference PHYSOR 2016: Unifying Theory and Experiments in the 21st Century, DOI:10.1016/j.pnucene.2017.06.013

[8] T. Downar, Y. Xu, V. Seker. PARCS v3.0 U.S. NRC Core Neutronics Simulator - Theory Manual. RES/U.S. NRC, Rockville, Md, 2012.

[9] B. T. Rearden, M. Jessee. SCALE Code System. ORNL/TM-2005/39, 2016.

[10] J. Leppänen. Serpent - a Continous-energy Monte Carlo Reactor Physics Burnup Calculation Code. VTT Technical Research Centre of Finland, 2015.

[11] Purdue advanced reactor core simulator. Purdue University Accessed: 2019-05-14. https://engineering.purdue.edu/PARCS

[12] B. T. Rearden, M. Jessee. SCALE Code System. ORNL/TM-2005/39, 2018.

[13] A. Ward, T. Downar, Y. Xu. GenPMAXS v6.2 Code for Generation the PARCS Cross Section Interface File PMAXS. RES/U.S. NRC, Rockville, Md, 2016. http: //nuram.engin.umich.edu/software/genpmaxs/ 